Interfaces and Free Boundaries 12 (2010), 75-84

DOI $10.4171 / \mathrm{IFB} / 227$

\title{
Convexity breaking of the free boundary for porous medium equations
}

\author{
KAZUHIRO ISHIGE \\ Mathematical Institute, Tohoku University, \\ Aoba, Sendai 980-8578, Japan \\ E-mail: ishige@math.tohoku.ac.jp \\ PAOLO SALANI \\ Dipartimento di Matematica 'U. Dini', \\ Viale Morgagni 67/A, 50137 Firenze, Italy \\ E-mail: salani@math.unifi.it
}

[Received 1 December 2008 and in revised form 15 December 2009]

We investigate the preservation of convexity of the free boundary by the solutions of the porous medium equation. We prove that starting with an initial datum with some kind of suboptimal $\alpha$ concavity property, the convexity of the positivity set can be lost in a short time.

\section{Introduction}

We consider the Cauchy problem for the porous medium equation,

$$
\begin{cases}\partial_{t} u=\Delta u^{m} & \text { in } \mathbb{R}^{N} \times(0, \infty), \\ u(x, 0)=\varphi(x) \geqslant 0 & \text { in } \mathbb{R}^{N},\end{cases}
$$

where $\partial_{t}=\partial / \partial t, N \geqslant 2, m>1$, and $\varphi \in C_{0}\left(\mathbb{R}^{N}\right)$. It is well known that the problem 1.1 has a unique strong solution $u \in C\left(\mathbb{R}^{N} \times(0, \infty)\right)$, and that the positivity set of $u(\cdot, t)$,

$$
P_{\varphi}(t)=\left\{x \in \mathbb{R}^{N}: u(x, t)>0\right\}
$$

is bounded for any $t \geqslant 0$ and increases with time.

This paper is concerned with the following problem.

Problem (P) Let $\Omega$ be a bounded convex domain and let the initial datum $\varphi$ be a nonnegative function, belonging to $C_{0}\left(\mathbb{R}^{N}\right) \cap C^{\infty}(\Omega)$, whose positivity set $P_{\varphi}(0)$ coincides with $\Omega$. Moreover, set $f=\varphi^{m-1}$ and assume that

$$
\left(A_{\alpha}\right)\left\{\begin{array}{l}
\text { (i) there exists a positive constant } \mu \text { such that } f+|\nabla f| \geqslant \mu \text { in } \Omega ; \\
\text { (ii) } f \text { is } \alpha \text {-concave in } \mathbb{R}^{N} \text { for some } \alpha \in[-\infty, \infty] .
\end{array}\right.
$$

Is then $P_{\varphi}(t)$ convex for every $t>0$ ? (See Section 2 for the definition of $\alpha$-concavity.)

The porous medium equation provides a simple model in many physical situations, in particular, the flow of an isentropic gas through a porous medium; in such a case, $u$ and $u^{m-1}$ represent the density and the pressure of the gas, respectively. Due to its practical interest, regularity and geometric properties of the free boundary $\partial P_{\varphi}(t)$ have been extensively studied by many 
mathematicians; see for instance [1], [3]-[8], [14]-[16] and the monograph [17], which gives a good survey of the study of the porous medium equations and a comprehensive list of references. In connection to problem (P), in [14], Lee and Vázquez proved that $P_{\varphi}(t)$ is convex for all sufficiently large $t$, even if $P_{\varphi}(0)$ is not convex; in fact, they proved that the pressure $u^{m-1}$ becomes a concave function on its support after a suitably long time, if the initial support is compact. Furthermore, under suitable regularity conditions on the initial datum $\varphi$, in [7], Daskalopoulos, Hamilton, and Lee proved that, if $f=\varphi^{m-1}$ satisfies condition $\left(A_{\alpha}\right)$ with $\alpha=1 / 2$, then the pressure $u(\cdot, t)^{m-1}$ remains $1 / 2$-concave for $t>0$, which again implies that the set $P_{\varphi}(t)$ is convex for every $t>0$. This gives an affirmative answer to problem (P) with $\alpha \geqslant 1 / 2$. We may then wonder if the exponent $1 / 2$ has some optimality property and, similarly to [14] and [17, page 520], we ask: is the $\alpha$ concavity of the initial pressure $u^{m-1}$ preserved even if $\alpha<1 / 2$ ? And, if not, does the $\alpha$-concavity $(\alpha<1 / 2)$ of the initial datum implies the $\beta$-concavity of $u(\cdot, t)$ for every $t>0$, for some suitable $\beta<\alpha$ ? Notice that problem (P) corresponds exactly to the latter question for $\beta=-\infty$.

In this paper we give a negative answer to problem $(\mathrm{P})$ (and hence to all the stronger questions posed above) for some $\alpha>0$, proving the following result.

THEOREM 1.1 Let $\Omega$ be a $C^{2}$ bounded convex domain in $\mathbb{R}^{N}, N \geqslant 2$, and let $t_{*}>0$. Then there exists a nonnegative function $\varphi \in C_{0}\left(\mathbb{R}^{N}\right)$ satisfying the condition $\left(A_{\alpha}\right)$ for some $\alpha>0$ and such that $P_{\varphi}(0)=\Omega$, while $P_{\varphi}(t)$ is not convex for some $t \in\left(0, t_{*}\right)$.

The above theorem essentially tells us that even starting with an initial datum with some kind of suboptimal concavity, the spatial convexity of the free boundary can be lost in an (arbitrarily chosen) short time. We remark that we are not simply saying that the $\alpha$-concavity of the initial pressure (for some $\alpha<1 / 2$ ) is not preserved, but that such a property may be completely destroyed by diffusion in a porous medium, since an $\alpha$-concave initial datum can result in a solution which is not even quasi-concave at any time $t>0$.

Notice that quasi-concavity is the weakest concavity property one can imagine: roughly speaking, a function $u$ is quasi-concave if all its superlevel sets are convex. In fact, this corresponds to $(-\infty)$-concavity.

We finally recall that $\alpha$-concavity for all $\alpha>0$ implies log-concavity; hence Theorem 1.1 shows in particular that starting with a log-concave initial datum is not sufficient to maintain the convexity of $P_{\varphi}(t)$. For comparison, we recall that the heat flow, corresponding to $(1.1)$ for $m=1$, preserves log-concavity (see [2], [13], [10]). In this connection, the present authors proved in [9] that the mere quasi-concavity of the initial datum is not inherited, in general, by the solutions of the heat equation. Now, we are able to improve this result by showing that $\alpha$-concavity, for some $\alpha<0$, is not necessarily preserved by the heat flow. Indeed, in Theorem 4.1, we construct examples of $\alpha$-concave initial data, with $\alpha<0$, that generate a heat distribution which is not quasi-concave after a small time.

\section{Preliminaries and notation}

In this section we introduce some notation and recall some basic properties of $\alpha$-concave functions and of solutions of the porous medium equation.

For any $x \in \mathbb{R}^{N}$ and $r>0$, we put $B(x, r)=\left\{y \in \mathbb{R}^{N}:|x-y|<r\right\}$. Let $D$ be a set in $\mathbb{R}^{N}$. We denote by $\chi_{D}$ the characteristic function of $D$, that is, $\chi_{D}(x)=1$ for $x \in D$ and $\chi_{D}(x)=0$ for $x \notin D$. 
Let $\lambda \in(0,1)$ and $\alpha \in[-\infty, \infty]$; for $s, t \geqslant 0$ with $s t>0$ we define

$$
g_{\alpha}(s, t: \lambda)= \begin{cases}\left(\lambda s^{\alpha}+(1-\lambda) t^{\alpha}\right)^{1 / \alpha} & \text { if } \alpha \notin\{ \pm \infty, 0\}, \\ \min \{s, t\} & \text { if } \alpha=-\infty, \\ \max \{s, t\} & \text { if } \alpha=\infty, \\ s^{\lambda} t^{1-\lambda} & \text { if } \alpha=0,\end{cases}
$$

and

$$
g_{\alpha}(s, t: \lambda)=0 \quad \text { if } s t=0 .
$$

Let $u$ be a nonnegative function defined in a convex set $\Omega$. Following [2] and [11], we say that $u$ is $\alpha$-concave if

$$
u((1-\lambda) x+\lambda y) \geqslant g_{\alpha}(u(x), u(y): \lambda)
$$

for all $x, y \in \Omega$ and $\lambda \in[0,1]$.

In other words, for $\alpha>0[<0], u$ is quasi-concave if $u^{\alpha}$ is positive and concave [convex] in a convex set $P_{u}$ and vanishes outside $P_{u}$. Furthermore, $u$ is 0 -concave (or log-concave) if $u$ is positive in a convex set $P_{u}$, vanishes outside $P_{u}$ and $\log u$ is concave in $P_{u}$.

For more details regarding $\alpha$-concave functions, we refer to [2] and [11]; here we recall just the following two properties, which are used in this paper:

(C1) if $f$ is $\alpha$-concave, then $f$ is $\beta$-concave for all $\beta \leqslant \alpha$.

(C2) Let $\alpha, \beta \in[0, \infty]$, and $f$ and $g$ be $\alpha$-concave and $\beta$-concave functions on bounded convex subsets $\Omega_{1}$ and $\Omega_{2}$ of $\mathbb{R}^{N}$, respectively. Then the convolution $f * g$, defined as usual by

$$
(f * g)(x)=\int_{\Omega_{1} \cap\left(x-\Omega_{2}\right)} f(y) g(x-y) \mathrm{d} y,
$$

is $\gamma$-concave in $\Omega_{1}+\Omega_{2}$, with $\gamma^{-1}=N+\alpha^{-1}+\beta^{-1}$.

Next we recall some properties of solutions of 1.1 . For any nonnegative function $\varphi \in L^{1}\left(\mathbb{R}^{N}\right)$, there exists a unique (strong) solution $u=S(t) \varphi$ of 1.1], and the following statements hold (see Section 9 in [17]):

(S1) $(S(t) \varphi)(x)$ is a continuous function in $\mathbb{R}^{N} \times(0, \infty)$.

(S2) If $0 \leqslant \varphi_{1} \leqslant \varphi_{2}$ almost everywhere in $\mathbb{R}^{N}$ and $\varphi_{2} \in L^{1}\left(\mathbb{R}^{N}\right)$, then

$$
0 \leqslant\left(S(t) \varphi_{1}\right)(x) \leqslant\left(S(t) \varphi_{2}\right)(x) \quad \text { in } \mathbb{R}^{N} \times(0, \infty) .
$$

(S3) If $P_{\varphi}(0)$ is bounded, then $P_{\varphi}(t)$ is bounded for all $t>0$ and

$$
P_{\varphi}\left(t_{1}\right) \subset P_{\varphi}\left(t_{2}\right) \quad \text { if } 0<t_{1}<t_{2} .
$$

It is well known that the equation (1.1) has a family of self-similar solutions called Barenblatt-Pattle solutions given by

$$
U_{M}(x, t)=t^{-k N}\left[c_{M}-\frac{(m-1) k}{2 m}|x|^{2} t^{-2 k}\right]_{+}^{1 /(m-1)},
$$

where $[v]_{+}=\max \{v, 0\}, k=(N(m-1)+2)^{-1}$ and $c_{M}$ is given by mass conservation

$$
\int_{\mathbb{R}^{N}} U_{M}(x, t) \mathrm{d} x=M \geqslant 0 .
$$


Then, for any $t>0$, we have

$$
\left\{x \in \mathbb{R}^{N}: U_{M}(x, t)>0\right\}=\left\{x \in \mathbb{R}^{N}:|x|<c_{M}^{\prime} t^{k}\right\},
$$

where $c_{M}^{\prime}=\sqrt{2 m c_{M} /(m-1) k}$.

The following proposition, taken from [4], gives a lower bound of the speed of propagation of the support of solutions for porous medium equations.

Proposition 2.1 (See Proposition 3.1 in [4]) Let $\varphi \in L^{1}\left(\mathbb{R}^{N}\right)$ be a nonnegative function and set

$$
E(x: \varphi)=\sup _{R>0} R^{-\left(N+\frac{2}{m-1}\right)} \int_{|y-x|<R} \varphi(y) \mathrm{d} y .
$$

There exists a constant $c_{*}=c_{*}(N, m)$ such that $(S(t) \varphi)(x)=0$ for every $(x, t) \in \mathbb{R}^{N} \times(0, \infty)$ such that

$$
0<t<c_{*} E(x: \varphi)^{1-m} .
$$

\section{Proof of Theorem 1.1}

Let $\Omega$ be a $C^{2}$ bounded convex domain in $\mathbb{R}^{N}$. Here we can assume, without loss of generality, that

$$
\Omega \subset\left\{x_{N}<0\right\}, \quad 0 \in \partial \Omega
$$

For any $r>0$, put

$$
\Omega(r)=\{x \in \Omega: \operatorname{dist}(x, \partial \Omega)>r\} .
$$

Then there exists a positive constant $\delta$ such that

$$
\bigcup_{x \in \Omega(r)} B(x, r)=\Omega, \quad 0<r<\delta .
$$

For $n=1,2, \ldots$, define

$$
\Omega_{n}=n \Omega, \quad \Omega_{n}^{\prime}=n \Omega\left(n^{-2}\right), \quad \Omega_{n}^{\prime \prime}=n \Omega\left(2 n^{-2}\right)
$$

By (3.1), the convexity and the regularity of $\Omega$, we have

$$
\Omega_{n} \subset \Omega_{n+1} \subset\left\{x_{N}<0\right\}, \quad \bigcup_{n=1}^{\infty} \Omega_{n}=\bigcup_{n=1}^{\infty} \Omega_{n}^{\prime}=\bigcup_{n=1}^{\infty} \Omega_{n}^{\prime \prime}=\left\{x_{N}<0\right\} .
$$

Set

$$
\rho(x)= \begin{cases}1-|x|^{2} & \text { for }|x|<1 \\ 0 & \text { for }|x| \geqslant 1 .\end{cases}
$$

Furthermore, for any $n=1,2, \ldots$, we put

$$
\rho_{n}(x)=n^{N} \rho(n x), \quad d_{n}(x)=\int_{\Omega_{n}^{\prime}} \rho_{n}(x-y) \mathrm{d} y .
$$

Then, by 3.2, we have

$$
\left\{x \in \mathbb{R}^{N}: d_{n}(x)>0\right\}=\Omega_{n}
$$


for all $n=1,2, \ldots$ with $n>\delta^{-1}$. Furthermore, since $\rho$ is 1 -concave, by (C2) the function $d_{n}$ is $(N+1)^{-1}$-concave in $\mathbb{R}^{N}$. This implies that $d_{n}^{1 /(N+1)}$ is concave in $\mathbb{R}^{N}$. Therefore, since $d_{n}(x)=1$ in $\Omega_{n}^{\prime \prime}$ and $d_{n}(x)=0$ on $\partial \Omega_{n}$, for any sufficiently large $n$ there exists a constant $c_{n, 1}$ such that

$$
d_{n}(x)^{1 /(N+1)} \geqslant c_{n, 1} \operatorname{dist}\left(x, \partial \Omega_{n}\right) \quad \text { in } \Omega_{n} \backslash \Omega_{n}^{\prime \prime} .
$$

Let $A$ be a positive constant to be chosen later. Put

$$
\varphi_{n}(x)=\left(A \int_{\Omega_{n}^{\prime}} e^{-|y|^{2}} \rho_{n}(x-y) \mathrm{d} y\right)^{1 /(N+1)(m-1)}
$$

and $u_{n}(x, t)=\left(S(t) \varphi_{n}\right)(x)$. For any sufficiently large $n$, since $e^{-|x|^{2}} \chi_{\Omega_{n}^{\prime}}$ is $\alpha_{n}$-concave for some $\alpha_{n}>0$, by (C2) there exists a positive constant $\beta_{n}$ such that

$$
\text { the function } \varphi_{n}^{m-1} \text { is } \beta_{n} \text {-concave in } \mathbb{R}^{N} \text {. }
$$

On the other hand, by $3.2-3.5$, for $n>\delta^{1 / 2}$, we have

$$
\left\{\varphi_{n}(x)>0\right\}=\bigcup_{x \in \Omega_{n}^{\prime}} B\left(x, n^{-1}\right)=n \bigcup_{x \in \Omega\left(n^{-2}\right)} B\left(x, n^{-2}\right)=n \Omega .
$$

Furthermore, by (3.5)-3.7), for any sufficiently large $n$, there exists a constant $c_{n, 2}$ such that

$$
\varphi_{n}(x)^{m-1} \geqslant\left(A c_{n, 2} d_{n}(x)\right)^{1 /(N+1)} \geqslant c_{n, 1}\left(A c_{n, 2}\right)^{1 /(N+1)} \operatorname{dist}\left(x, \partial \Omega_{n}\right)
$$

in $\Omega_{n} \backslash \Omega_{n}^{\prime \prime}$. This implies that there exist positive constants $c_{n, 3}$ and $\delta_{n}$ such that

$$
\left|\nabla\left(\varphi_{n}(x)^{m-1}\right)\right| \geqslant c_{n, 3}
$$

for all $x \in \Omega_{n}$ with $\operatorname{dist}\left(x, \partial \Omega_{n}\right)<\delta_{n}$.

Let $t_{*}>0$. By [2.2], there exists a positive constant $L$ such that

$$
0<t_{*}<c_{*} E\left(L e_{N}: \chi_{\left\{x_{N}<0\right\}}\right)^{1-m},
$$

where $c_{*}$ is the constant given in Proposition 2.1. Since $c_{M}^{\prime} \rightarrow \infty$ as $M \rightarrow \infty$ we can take a sufficiently large constant $M$ such that

$$
c_{M}^{\prime}+L+2<c_{M}^{\prime}\left(t_{*}+1\right)^{k} .
$$

Since

$$
\operatorname{supp} U_{M}\left(\cdot+\left(c_{M}^{\prime}+2\right) e_{N}, 1\right) \subset B\left(-\left(c_{M}^{\prime}+2\right) e_{N}, c_{M}^{\prime}\right) \subset\left\{x_{N}<-1\right\},
$$

by (3.3) and 3.9) we can take a sufficiently large constant $A$ so that

$$
\varphi_{n}(x) \geqslant U_{M}\left(x+\left(c_{M}^{\prime}+2\right) e_{N}, 1\right) \quad \text { in } \mathbb{R}^{N}
$$

for all sufficiently large $n$. Then, by (S2) and (3.13), we have

$$
u_{n}(x, t) \geqslant U_{M}\left(x+\left(c_{M}^{\prime}+2\right) e_{N}, t+1\right) \quad \text { in } \mathbb{R}^{N} \times(0, \infty),
$$


and by 2.1 and 3.12 there exists a positive constant $L^{\prime}>L$ such that

$$
u_{n}\left(l e_{N}, t_{*}\right) \geqslant U_{M}\left(\left(l+c_{M}^{\prime}+2\right) e_{N}, t_{*}+1\right)>0
$$

for all $0<l \leqslant L^{\prime}$ and sufficiently large $n$.

On the other hand, since

$$
\sup _{n \in \mathbb{N}}\left\|\varphi_{n}\right\|_{L^{\infty}\left(\mathbb{R}^{N}\right)}<\infty, \quad \lim _{|x| \rightarrow \infty} \varphi_{n}(x)=0 \quad \text { uniformly for all } n,
$$

by (3.3) and (3.9) there exists a constant $R_{*}$ such that

$$
E\left(x: \varphi_{n}\right)=R^{-\left(N+\frac{2}{m-1}\right)} \sup _{x \in \mathbb{R}^{N}} \int_{|y-x|<R} \varphi_{n}(y) \mathrm{d} y \leqslant E\left(L e_{N}: \chi_{\left\{x_{N}<0\right\}}\right)
$$

for all $x=\left(x^{\prime}, x_{N}\right)$ with $x^{\prime} \geqslant R_{*}$ and $x_{N} \geqslant L$ and for all $n \in \mathbb{N}$. By Proposition 2.1, 3.11) and (3.15), we have

$$
u_{n}\left(x, t_{*}\right)=0
$$

for all $x=\left(x^{\prime}, x_{N}\right)$ with $x^{\prime} \geqslant R_{*}$ and $x_{N} \geqslant L$ and all $n \in \mathbb{N}$.

Put

Then $\lambda<1$ and

$$
\lambda=\frac{L+L^{\prime}+2}{2\left(L^{\prime}+1\right)} .
$$

$$
1-\lambda=\frac{L^{\prime}-L}{2\left(L^{\prime}+1\right)} .
$$

By (3.3), we can take a large integer $n$ so that (3.14) holds and

$$
\bar{x}=\left(\frac{R_{*}}{1-\lambda}, 0, \ldots, 0,-1\right) \in n \Omega,
$$

and fix $n$. Then, by (3.9) and 3.17), we have

$$
u_{n}(\bar{x}, t)>0 \text { for all } t>0 .
$$

Furthermore, the set $P_{\varphi_{n}}\left(t_{*}\right)$ is not convex. Indeed, if $P_{\varphi_{n}}\left(t_{*}\right)$ is convex, then, by (3.14), (3.17), and 3.18, we have

$$
(1-\lambda) \bar{x}+\lambda\left(0, \ldots, 0, L^{\prime}\right)=\left(R_{*}, 0, \ldots, 0,\left(L+L^{\prime}\right) / 2\right) \in P_{\varphi_{n}}\left(t_{*}\right) .
$$

Since $\left(L+L^{\prime}\right) / 2>L$, this contradicts 3.16.

Finally, we put

$$
\varphi(x)=n^{N} \varphi_{n}(n x), \quad U(x, t)=n^{N} u_{n}\left(n x, n^{1 / k} t\right), \quad \bar{t}=n^{-1 / k} t_{*} \in\left(0, t_{*}\right),
$$

where $k=(N(m-1)+2)^{-1}$. Then $U(t)=S(t) \varphi$, and by $3.8, \varphi^{m-1}$ is $\beta_{n}$-concave in $\mathbb{R}^{N}$. Furthermore, by (3.9) and (3.10), we have $P_{\varphi}(0)=\Omega$ and

$$
\varphi(x)^{m-1}+\left|\nabla \varphi(x)^{m-1}\right| \geqslant C \quad \text { in } \Omega
$$

for some constant $C$. Therefore, since $P_{\varphi}(\bar{t})=n^{-1} P_{\varphi_{n}}\left(t_{*}\right)$ is not convex, the proof of Theorem 1.1 is complete. 


\section{Heat equation}

In this section we apply the argument of Section 3 to the heat equation, and improve a result of our previous paper [9]. Let $u$ be a nonnegative solution of

$$
\begin{cases}\partial_{t} u=\Delta u & \text { in } \Omega \times(0, \infty), \\ u(x, t)=0 & \text { on } \partial \Omega \times(0, \infty) \text { if } \partial \Omega \neq \emptyset, \\ u(x, 0)=\varphi(x) \geqslant 0 & \text { in } \Omega\end{cases}
$$

where $\Omega$ is a convex smooth domain in $\mathbb{R}^{N}$ and $N \geqslant 2$. Then, for any nonnegative solution $u$ of the Cauchy-Dirichlet problem (4.1), if the initial datum $u(\cdot, 0)$ is 0 -concave, then for any $t>0$, the solution $u(\cdot, t)$ is 0 -concave, in particular, $u(\cdot, t)$ is quasi-concave. In our previous paper [9], we discussed the preservation of the quasi-concavity by the heat flow, and gave an example of a quasi-concave initial datum for which the solution of 4.1) is not quasi-concave at some time. By the arguments in the previous sections and [9], we can now prove the following theorem.

THEOREM 4.1 Let $\Omega$ be any smooth convex domain in $\mathbb{R}^{N}$ (possibly $\Omega=\mathbb{R}^{N}$ ) and $t_{*}>0$. Then there exists a nonnegative function $\varphi \in C_{0}(\Omega)$ such that

(1) $\varphi$ is $\alpha$-concave in $\Omega$ for some $\alpha \in(-\infty, 0)$,

(2) the solution of 4.1) is not quasi-concave in $\Omega$ for some $t \leqslant t_{*}$.

Proof. Let

$$
\begin{aligned}
\psi(x) & =\left(1+e^{-|x|^{2}}\right) \chi_{\left\{x_{N}<0\right\}}, \\
v(x, t) & =\left(e^{t \Delta} \psi\right)(x) \equiv(4 \pi t)^{-N / 2} \int_{\mathbb{R}^{N}} \exp \left(-\frac{|x-y|^{2}}{4 t}\right) \psi(y) \mathrm{d} y .
\end{aligned}
$$

Let $t_{*}>0$. Then, by the same argument as in Lemma 3.1 of [9], there exist two points $X$ and $Y$ in $\mathbb{R}^{N}$ such that

$$
v\left(X, t_{*}\right)>\frac{1}{2}, \quad v\left(Y, t_{*}\right)>\frac{1}{2}, \quad v\left(\frac{X+Y}{2}, t_{*}\right)<\frac{1}{2} .
$$

Next, for any $n=1,2, \ldots$, we put

$$
\psi_{n}(x)=\psi(x) \chi_{B\left(x_{n}, n\right)}(x), \quad x_{n}=-\left(n+n^{-1}\right) e_{N}, \quad u_{n}(x, t)=\left(e^{t \Delta} \psi_{n}\right)(x) .
$$

Then we have

$$
\lim _{n \rightarrow \infty} \sup _{x \in B(0, R)}\left|u_{n}(x, t)-v(x, t)\right|=0
$$

for any $R>0$ and $t>0$, and by 4.2), for any sufficiently large $n$,

$$
u_{n}\left(X, t_{*}\right)>\frac{1}{2}, \quad u_{n}\left(Y, t_{*}\right)>\frac{1}{2}, \quad u_{n}\left(\frac{X+Y}{2}, t_{*}\right)<\frac{1}{2} .
$$

We take a sufficiently large $n$ so that 4.3 holds, and fix $n$. Put

$$
\eta_{m}(x)= \begin{cases}0 & \text { if }\left|x-x_{n}\right|<n-m^{-1}, \\ \left(n-\left|x-x_{n}\right|\right)^{-1}-m & \text { if } n-m^{-1} \leqslant\left|x-x_{n}\right|<n,\end{cases}
$$



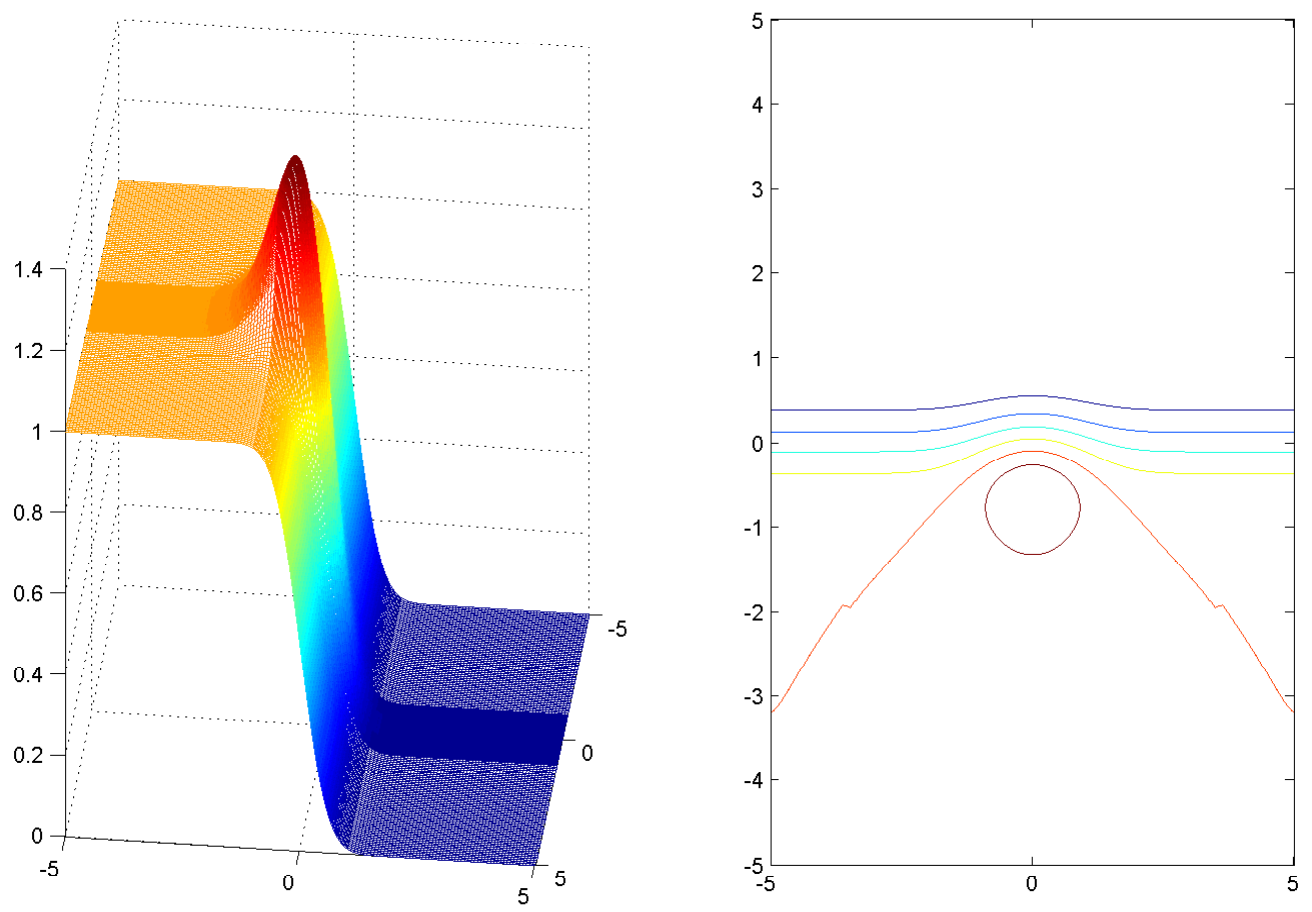

FIG. 1. The function $v$ and some of its level sets at time $t=0.1$.

where $m=1,2, \ldots$ Then $\eta_{m}(x)$ is a convex function in $B\left(x_{n}, n\right)$. On the other hand, since $\psi_{n}$ is $\alpha_{n}$-concave for some $\alpha_{n}<0, \psi_{n}^{\alpha_{n}}$ is convex in $B\left(x_{n}, n\right)$, and the function $\psi_{n}^{\alpha_{n}}+\eta_{m}$ is also convex in $B\left(x_{n}, n\right)$. Put

$$
\begin{aligned}
& \varphi_{m}(x)= \begin{cases}\left(\psi_{n}(x)^{\alpha_{n}}+\eta_{m}(x)\right)^{1 / \alpha_{n}} & \text { if } x \in B\left(x_{n}, n\right), \\
0 & \text { otherwise, }\end{cases} \\
& u_{m}(x, t)=\left(e^{t \Delta} \varphi_{m}\right)(x) .
\end{aligned}
$$

Then $\varphi_{m}(x)$ is an $\alpha_{n}$-concave function such that

$$
\begin{aligned}
& \varphi_{m} \in C_{0}\left(\mathbb{R}^{N}\right), \quad \operatorname{supp} \varphi_{m}=\overline{B\left(x_{n}, n\right)} \subset\left\{x_{N}<-n^{-1}\right\}, \\
& \lim _{m \rightarrow \infty} \sup _{x \in B\left(x_{n}, n\right)}\left\|\varphi_{m}(x)-\psi_{n}(x)\right\|=0 .
\end{aligned}
$$

Hence

$$
\lim _{m \rightarrow \infty} \sup _{B(0, R)}\left|u_{m}(x, t)-u_{n}(x, t)\right|=0
$$

for any $R>0$ and $t>0$. This together with (4.3) implies that, for any sufficiently large $m$,

$$
u_{m}\left(X, t_{*}\right)>\frac{1}{2}, \quad u_{m}\left(Y, t_{*}\right)>\frac{1}{2}, \quad u_{m}\left(\frac{X+Y}{2}, t_{*}\right)<\frac{1}{2}
$$

thus $u_{m}\left(\cdot, t_{*}\right)$ is not quasi-concave in $\mathbb{R}^{N}$. 
If $\Omega=\mathbb{R}^{N}$, we put $\varphi(x)=\varphi_{m}(x)$, and the proof of Theorem 4.1 is complete.

For $\Omega \neq \mathbb{R}^{N}$, without loss of generality, we can assume 3.1 . We pick a sufficiently large $m$ so that (4.5) holds. By (3.3) and 4.4, we can take an integer $l$ such that $\varphi_{m} \in C_{0}\left(\Omega_{l}\right)$. Let $U_{l}$ be the solution of

$$
\begin{cases}\partial_{t} U=\Delta U & \text { in } \Omega_{l} \times(0, \infty) \\ U(x, t)=0 & \text { on } \partial \Omega_{l} \times(0, \infty), \\ U(x, 0)=\varphi_{m}(x) & \text { in } \Omega_{l}\end{cases}
$$

Then

$$
\lim _{m \rightarrow \infty} \sup _{B(0, R)}\left|U_{l}(x, t)-u_{m}(x, t)\right|=0
$$

for any $R>0$ and $t>0$. By 4.5 , there exists a sufficiently large $L$ such that

$$
U_{L}\left(X, t_{*}\right)>\frac{1}{2}, \quad U_{L}\left(Y, t_{*}\right)>\frac{1}{2}, \quad U_{L}\left(\frac{X+Y}{2}, t_{*}\right)<\frac{1}{2} ;
$$

thus $U_{L}\left(\cdot, t_{*}\right)$ is not quasi-concave in $\Omega_{L}$. Finally, we put

$$
u(x, t)=U_{L}\left(L x, L^{2} t\right), \quad \varphi(x)=\varphi_{m}(L x) \in C_{0}(\Omega)
$$

for all $x \in \Omega$ and $t>0$. Then $u$ is a solution of $(1.1)$ and $\varphi$ is $\alpha_{n}$-concave in $\Omega$. Furthermore, by 4.7$), u(\cdot, \bar{t})$ is not quasi-concave in $\Omega$, for $\bar{t}=L^{-2} t_{*} \leqslant t_{*}$; thus the proof of Theorem 4.1 is complete.

\section{Acknowledgements}

The main part of this work was completed while the second author was visiting the first one at Tohoku University. The visit was supported by the Grant-in-Aid for Scientific Research (B) (No. 19340036), Japan Society for the Promotion of Science.

\section{REFERENCES}

1. Aronson, D. G., \& VÁzQuez, J. L. Eventual $C^{\infty}$-regularity and concavity for flows in onedimensional porous media. Arch. Ration. Mech. Anal. 99 (1987), 329-348. Zbl 0642.76108 MR 0898714

2. Brascamp, H. J., \& Lieb, E. H. On extensions of the Brunn-Minkowski and Prékopa-Leindler theorems, including inequalities for $\log$ concave functions, and with an application to the diffusion equation. J. Funct. Anal. 22 (1976), 366-389. Zbl 0334.26009 MR 0450480

3. Caffarelli, L. A., \& Friedman, A. Regularity of the free boundary of a gas flow in an $n$-dimensional porous medium. Indiana Univ. Math. J. 29 (1980), 361-391. Zbl $0439.76085 \mid$ MR 0570687

4. Caffarelli, L. A., Vázquez, J. L., \& Wolanski, N. I. Lipschitz continuity of solutions and interfaces of the $N$-dimensional porous medium equation. Indiana Univ. Math. J. 36 (1987), 373-401. Zbl 0644.35058 MR 0891781

5. Daskalopoulos, P., \& Hamilton, R. The free boundary for the $n$-dimensional porous medium equation. Int. Math. Res. Notices 1997, no. 17, 817-831. Zbl 0886.35114 MR 1474840

6. Daskalopoulos, P., \& Hamilton, R. Regularity of the free boundary for the porous medium equation. J. Amer. Math. Soc. 11 (1998), 899-965. Zbl 0910.35145 MR 1623198

7. Daskalopoulos, P., Hamilton, R., \& LeE, K. All time $C^{\infty}$-regularity of the interface in degenerate diffusion: a geometric approach. Duke Math. J. 108 (2001), 295-327. Zbl 1017.35052 MR 1833393 
8. Galaktionov, V. A., \& VÁzQuez, J. L. Geometrical properties of the solutions of one-dimensional nonlinear parabolic equations. Math. Ann. 303 (1995), 741-769. Zbl 0842.35006 MR 1359958

9. Ishige, K., \& SAlani, P. Is quasi-concavity preserved by heat flow? Arch. Math. (Basel) 90 (2008), 450-460. Zbl 1176.35012 MR 2414248

10. Greco, A., \& KAWOHL, B. Log-concavity in some parabolic problems. Electron. J. Differential Equations 1999, no. 19, 12 pp. Zbl 0921.35030 MR 1692565

11. Kennington, A. U. Power concavity and boundary value problems. Indiana Univ. Math J. 34 (1985), 687-704. Zbl 0549.35025 MR 0794582

12. Kennington, A. U. Convexity of level curves for an initial value problem. J. Math. Anal. Appl. 133 (1988), 324-330. Zbl 0696.35080 MR 0954709

13. KorevaAR, N. J. Convex solutions to nonlinear elliptic and parabolic boundary value problems. Indiana Univ. Math. J. 32 (1983), 603-614. Zbl 0481.35024 MR 0703287

14. LEE, K.-A., \& VÁZQUEZ, J. L. Geometrical properties of solutions of the porous medium equation for large times. Indiana Univ. Math. J. 52 (2003), 991-1016. Zbl 1080.35037|| MR 2001942

15. LeE, K.-A., \& VÁzQuez, J. L. Parabolic approach to nonlinear elliptic eigenvalue problems. Adv. Math. 219 (2008), 2006-2028. Zbl 1152.35060 MR 2456273

16. NiCKEL, K. Gestaltaussagen über Lösungen parabolischer Differentialgleichungen. J. Reine Angew. Math. 211 (1962), 78-94. Zbl 0127.31801 MR 0146534

17. VÁzquez, J. L. The Porous Medium Equation. Mathematical Theory. Oxford Math. Monogr., Oxford Univ. Press, Oxford (2007). Zbl 1107.35003 MR 2286292 